\title{
¿Realizan los alumnos las tareas de intercambio oral de información en el aula AICLE?
}

\author{
Zoraida Horrillo Godino \\ UniversitatAutònoma de Barcelona, Barcelona, España
}

Articulo recibido el 17 de noviembre de 2010; versión final recibida el 14 de abril de 2011)

\begin{abstract}
Numerosos estudios prueban que la participación de los estudiantes de segundas lenguas en tareas orales en pequeños grupos promueve aprendizajes (lingüísticos). Sin embargo, los profesores se muestran reacios a implementar dichas tareas porque creen que los alumnos las abandonarán en cuanto les cedan el control de su ejecución. Para explorar qué hacen realmente los alumnos cuando controlan una tarea oral, este artículo estudia los dos tipos de actividad que toda una clase de Educación Secundaria Obligatoria desarrolla mientras trabaja en una tarea por parejas: la actividad orientada hacia la ejecución de dicha tarea (actividad on) y la que supone un abandono de la misma (actividad off). La tarea que se les asigna, un intercambio de información de ciencias naturales en inglés, fue diseñada a partir del enfoque AICLE (Aprendizaje Integrado de Contenidos y Lengua) y la técnica del rompecabezas. El tiempo que invierte cada díada en subtipos de actividad on y off se midió en segundos. Subsiguientemente se calcularon porcentajes. Un resultado destacable es el hecho de que algunos alumnos continuaron utilizando la lengua meta después de abandonar la tarea. Otro indica que casi todas las parejas invirtieron la mayor parte de su tiempo a conversar sobre el tema meta de ciencias naturales.
\end{abstract}

\section{Introducción}

Durante la última década del s. XX, la Unión Europea experimentó una explosión de iniciativas educativas multilingües que supuso la ruptura con los modelos de inmersión norteamericanos. Dichas iniciativas permitieron la creación de programas que tuviesen en cuenta realidades locales, regionales, nacionales e internacionales (Marsh, Maljiers y Hartiala 2001). Los programas multilingües evolucionaron así hacia el enfoque de enseñanza/aprendizaje AICLE (Aprendizaje Integrado de Contenidos y Lengua) (Ibíd. 2001), que consiste en utilizar una lengua segunda (L2) o extranjera (LE) como medio de instrucción para 
asignaturas como Matemáticas, Música o Historia, i.e. para asignaturas de contenido ${ }^{1}$. La mayoría de los países miembros de la U.E. ya ofrecen la posibilidad de utilizar ese enfoque en la educación del conjunto de su ciudadanía, aunque su uso aún no se ha generalizado (Eurydice 2006).

Numerosos estudios recientes prueban que el uso del enfoque AICLE en el contexto europeo puede ayudar a los alumnos a mejorar conocimientos y competencias relacionadas con asignaturas de contenido y con L2 o LE (p.ej. Hüttner y Rieder-Bünemann 2007; Linares y Whitaker 2007; Lyster 2007). Sin embargo, la novedad del enfoque hace necesario continuar estudiando el diseño y la evaluación de propuestas AICLE. Como contribución a ese campo, el equipo de investigación CLIL-SI ha desarrollado el proyecto de investigación competitiva y colaborativa ArtICLE ${ }^{2}$, al cual se adscribe el trabajo final de máster de la autora, Horrillo Godino (2006). De este trabajo deriva el presente estudio.

El objetivo de ArtICLE es diseñar tareas cooperativas de Ciencias Naturales (CCNN) en LE y evaluar su adecuación a aulas AICLE inclusivas catalanas, en las que la LE es la tercera lengua de instrucción. El presente estudio explora los dos tipos de actividad que una clase natural de tercer curso de Educación Secundaria Obligatoria desarrolla, por parejas, para llevar a cabo una tarea de intercambio de información oral en CCNN en inglés: la actividad orientada hacia la ejecución de dicha tarea y la actividad que supone un abandono de la misma.

Este estudio se ha realizado para aportar datos empíricos sobre la participación de todas las parejas de una clase de habilidades mixtas en tareas orales de intercambio de información. Aportar datos sobre toda una clase puede resultar de utilidad al profesorado, que teme que la implementación de ese tipo de tareas en su aula sea una pérdida de tiempo, porque la mayoría de las parejas dedicarían el tiempo asignado a la tarea a hacer alguna otra cosa sin valor pedagógico. 


\section{Marco teórico}

La concepción sociocultural del aprendizaje

La hipótesis de partida del proyecto ArtICLE es que la conversación entre aprendices, desencadenada a partir de tareas pedagógicas estructuradas, se conforma como un entorno favorecedor de aprendizajes lingüísticos y no lingüísticos en aulas AICLE inclusivas (Escobar y Nussbaum 2008). Esta hipótesis parte de una concepción sociocultural del aprendizaje (Lantolf 2006). Dicha concepción sostiene que la interacción social no es sólo el contexto del aprendizaje. La interacción está vinculada a la cognición mediante la actividad verbal situada (Mondada y Pekarek-Doehler 2004), que constituye un recurso básico para participar en las actividades del aula. La participación en esas actividades se convierte así en el proceso y el producto del aprendizaje (Zuengler y Miller 2006).

La participación en actividades AICLE que implican interacción verbal constituye una posibilidad para progresar en la adquisición de conocimientos relacionados con la lengua meta y la materia. Incluso los alumnos con problemas de aprendizaje pueden beneficiarse de la participación en dichas actividades (Jiménez 2009; Jiménez y Bazoco 2009). Escobar y Nussbaum (2008), usando parte del corpus del presente estudio, analizan la interacción de tres díadas con características muy diferentes. Su análisis revela que cada una de las díadas utiliza complejas estrategias de colaboración para convertir la tarea encomendada en un traje a medida para sus necesidades comunicativas e intereses particulares. La participación en este tipo de interacción dentro de una secuencia didáctica AICLE de CCNN, según Escobar y Sánchez (2009), mejora significativamente la fluidez y el repertorio léxico de los alumnos. Dicha mejora en la lengua conlleva la sofisticación de las competencias necesarias para producir el tipo de discurso académico con el que no sólo se transmiten los contenidos de CCNN, sino con el que se generan esos contenidos (Horrillo Godino 2008a, 2008b, 2009, 2010, 2011). 
Los estudios de Escobar y Nussbaum (2008) y Escobar y Sánchez (2009) mencionados en el párrafo anterior utilizan datos recogidos en aulas en las que se estaba realizando secuencias didácticas y tareas estructuradas según las técnicas del aprendizaje cooperativo. En la siguiente subsección se expone por qué estas técnicas resultan atractivas para las aulas AICLE inclusivas.

Aprendizaje cooperativo: la técnica del rompecabezas

Las técnicas de aprendizaje cooperativo generan un entorno en el que los alumnos miembros de pequeños grupos tienen que coordinar esfuerzos para completar una tarea (Kagan 1985; Watson 1992). Cuando estos descubren que sólo pueden conseguir su objetivo grupal, i.e. completar la tarea, si cada uno de los miembros del grupo logra su objetivo individual, se crea una interdependencia positiva de objetivos (Lew, Mesch, Johnson y Johnson 1986) que les obliga a ayudarse entre sí. En consecuencia, desde la perspectiva sociocultural, las técnicas de aprendizaje cooperativo resultan interesantes para las aulas inclusivas. También para las aulas AICLE, ya que en ellas se promueve, simultáneamente, la mejora de las competencias y habilidades sociales (Escobar y Nussbaum 2008) y de los conocimientos académicos (Slavin 1984).

El aprendizaje cooperativo siempre implica la existencia de una tarea estructurada y un incentivo a la cooperación (Watson 1992). La técnica del rompecabezas (Aronson, Blaney, Stephan, Sikes y Snapp 1975) está vinculada a la estructura de especialización de actividades. Cada miembro del grupo debe convertirse en el único experto en un subtema y, posteriormente, en el profesor que traspasa sus conocimientos a los demás miembros. Todos deben aprender sobre todos los subtemas para superar una prueba final en la que las puntuaciones individuales se combinan para obtener una puntuación definitiva basada en el éxito grupal. 


\section{Objetivo y preguntas}

A pesar de que se ha publicado pruebas del potencial beneficioso de las técnicas de aprendizaje cooperativo y de los enfoques AICLE y sociocultural, muchos docentes se muestran reacios a incluir en su programación tareas de intercambio oral de información entre expertos, ya que su implementación implica ceder el control de la misma a los alumnos. Los docentes no pueden, pues, saber a ciencia cierta si sus alumnos dedican el tiempo potencialmente asignado a la tarea a desarrollar alguna actividad encaminada a completarla (actividad on) o si, por el contrario, invierten ese tiempo en cualquier actividad que suponga un abandono de la misma (actividad off) ${ }^{4}$. Por este motivo, puede resultar de utilidad a los docentes conocer datos empíricos sobre el tiempo invertido en actividades on y off por parejas a las que se les ha pedido que lleven a cabo una tarea de intercambio oral de información sobre ciencias naturales en inglés. Este estudio pretende determinar si los alumnos de aulas AICLE inclusivas inician actividades on y las mantienen cuando se les asigna esa tarea.

Las preguntas asociadas al objetivo de este estudio son las siguientes:

1. ¿Cuánto tiempo invierten las parejas en la realización de la tarea?

2. ¿Durante cuánto tiempo mantienen una actividad on? ¿Y una actividad off?

3. Cuando las parejas interactúan en modo off, ¿utilizan la lengua meta? ¿Durante cuánto tiempo?

4. ¿Cuánto tiempo dedican las parejas a gestionar la subtarea, a conversar sobre el tema meta y a evaluar su propio rendimiento?

\section{Marco metodológico}

El concepto de tarea está en constante evolución, por lo que se han propuesto numerosas definiciones, p.ej. Long (1983), Nunan (1989) y Skehan (1998). Esas definiciones tienen dos puntos en común: la orientación hacia la obtención de un 
resultado y una finalidad comunicativa (Escobar 2004a) ${ }^{5}$. Dichas definiciones proporcionan al profesorado orientaciones sobre cómo diseñar tareas auténticas y significativas para el alumno, cómo seleccionarlas o cómo evaluarlas, aunque son conceptualizaciones tan abiertas que permiten la integración de tareas en propuestas didácticas diversas (Masats 2008).

Según Masats (2008), una de las definiciones pertenecientes al más reciente estadio de evolución del concepto de tarea es la de Bygate, Skehan y Swain (2001). Estos distinguen tres tipos de usuarios de tareas escolares o perspectivas émicas: la del profesor, la del investigador y la del alumno. Proponen una definición básica de tarea escolar y adaptaciones para los tres tipos de usuario. Su definición básica es la siguiente: “una tarea es una actividad que requiere que los educandos usen la lengua, poniendo énfasis en el significado, para alcanzar un objetivo” (Ibíd. 2001: 11). Su adaptación a la perspectiva del alumno es la siguiente:

una tarea es una actividad influenciada por elecciones de los educandos y susceptible de reinterpretación por parte de los mismos, la cual requiere que estos usen la lengua, poniendo énfasis en el significado, para alcanzar un objetivo. (Bygate, Skehan y Swain 2001: 11)

Esta definición es sensible al hecho de que los estudiantes adaptan las tareas a sus necesidades, como se ha observado en estudios empíricos (p. ej., Escobar y Nussbaum 2008).

La definición de tarea desde la perspectiva del alumno que ofrecen Bygate, Skehan y Swain (2001) es el punto de partida para la elaboración de la definición de actividad on durante el intercambio oral de información llevado a cabo por los alumnos participantes en este estudio. Más adelante se proporcionan detalles sobre la construcción de la mencionada definición y sobre su uso en este estudio. 


\section{Metodología}

Este estudio es el resultado de una investigación colaborativa, participante, en y para la acción docente que no pretende juzgar, sino describir e interpretar la interacción oral en un aula. Durante dicha investigación, se ha intentado proteger a los informantes mantenido su identidad real en el anonimato y ofreciéndoles la opción de no grabar su interacción o de destruir sus grabaciones in situ, si decidían no participar en la investigación una vez efectuada la grabación.

\section{Informantes}

Los informantes se dividen en alumnos y profesores. El grupo de profesores está formado por tres profesoras de inglés como LE. Una de ellas es la profesora habitual del grupo, mientras que las otras dos actúan como observadoras participantes.

El grupo de alumnos consiste en los veintitrés individuos que asistieron a clase el día que se recogió los datos. Estudian tercer curso de la ESO en un instituto público del área metropolitana de Barcelona, en el que se habla, mayoritariamente, catalán y español. También se habla el inglés, en las clases de LE.

El hecho de que los alumnos realizaran el intercambio de información objeto de estudio en díada permite que se les aluda como Pareja 1/Pareja 2/Pareja 3/ etc. La Pareja 4 es, en realidad, un trío, pero se mantiene la etiqueta pareja también para ellos en este estudio en pro de la simplicidad.

En la siguiente sección se detalla la aplicación de la técnica del rompecabezas al diseño de la tarea en la implementación de la cual se recogió los datos para este estudio.

\section{Actividades}

La tarea en la que se centra este estudio forma parte de Rainforests, una secuencia didáctica cuyo tema es la selva. Se escogió la selva porque es un tema central en el currículo de CCNN de la ESO (Escobar 2008). En el diseño de la secuencia ${ }^{3}$, 
trabajaron conjuntamente investigadoras expertas en el enfoque AICLE y profesoras de la ESO.

La secuencia didáctica Rainforests consiste en tres ciclos de tareas cooperativas (Escobar 2008). El primero comienza con la formación de parejas base, para reforzar la idea de que son un equipo. Seguidamente, se les comunica la finalidad escolar de la tarea 1, un test informal presentado como una competición. La puntuación final de cada pareja será la media de la obtenida por cada miembro, una medida con la que se pretende promover la percepción de la interdependencia de objetivos. A continuación, los alumnos realizan dos ejercicios introductorios. El objetivo del primero es activar sus conocimientos previos del tema. El objetivo del segundo es activar su conciencia metalingüística y adquirir cierta familiaridad con la lengua obligatoria para el contenido (Snow, Met y Genesee 1989; Gajo 2007), potencialmente problemática.

La tarea 1 se divide en dos subtareas. La primera comienza con la asignación a un miembro de las parejas base la etiqueta de Estudiante A y, al otro, Estudiante B. Todos los estudiantes A se reúnen para estudiar juntos un texto sobre la biodiversidad de la selva amazónica. Todos los B estudian material sobre la flora y la fauna en peligro de extinción en la misma zona. La segunda subtarea, foco de atención del presente estudio, coincide con la fase de intercambio oral de información en el seno de las parejas base, en las que uno de los miembros es experto en la biodiversidad amazónica y el otro, en especies en peligro de extinción. Al finalizar el intercambio, los alumnos realizan el test, completando, así, el ciclo de la tarea 1.

Las instrucciones para realizar la tarea 1 no sólo se proporcionan verbalmente al inicio de la misma. También por escrito, para que los alumnos puedan consultarlas en cualquier momento. La figura 1 contiene la traducción de las instrucciones escritas. 


\section{LA COMPETICIÓN RAINFORESTS}

Un estudiante es $A$. El otro es $B$.

$A$ y $B$ son un equipo.

FASE 1: Encuentra nuevos compañeros

1. Todos los estudiantes $A$ se sientan juntos y todos los estudiantes $B$ se sientan juntos.

FASE 2: Conviértete en un experto

2. Juntos, leed y escuchad. ¡Entended [lo que leéis!]

3. Juntos, aprended el contenido.

4. Practicad diciendo el contenido en voz alta.

5. Devolved vuestro texto al profesor y volved a vuestros asientos.

FASE 3: Enseña y aprende

6. El estudiante A escucha y aprende

7. El estudiante $B$ escucha y aprende

8. Juntos, revisad todo.

\section{¡LA COMPETICIÓN!}

Figura 1. Instrucciones para la realización de la tarea 1

\section{Corpus}

El corpus está compuesto por registros auditivos, una colección de notas de campo y los materiales utilizados para la implementación de la tarea 1. Cada grabación en audio recoge la actividad verbal de una pareja mientras realiza el intercambio oral de información. En total hay once grabaciones; una, por cada pareja. Las grabaciones constituyen la fuente principal de datos. Respecto a la colección de notas de campo, esta está formada por las anotaciones de las observadoras participantes. Dichas anotaciones se han utilizado para reconstruir los detalles del proceso de recolección de datos, incluyendo circunstancias particulares de la implementación de la tarea 1. En cuanto a los materiales, el contenido de las fotocopias con instrucciones para los alumnos y de los textos del Estudiante A y Estudiante $B$ se tuvo en cuenta durante la formulación de la definición de actividad on. 


\section{Recolección y procesamiento de datos}

Para iniciar la tarea 1, se pidió a los alumnos que formasen equipos uniéndose a la persona sentada a su lado, para obtener así parejas de habilidades mixtas. Después de hacer las actividades introductorias de la secuencia didáctica, se dio un magnetófono con una cinta etiquetada a cada pareja y se les pidió que, sin efectuar ninguna pausa, grabasen sus nombres, la fecha y la primera subtarea, el aprendizaje cooperativo de textos. Para grabar la segunda subtarea, el intercambio de información en díada, se escribieron en la pizarra unas reglas similares, traducidas al español en la figura 2:
1) Pulsad PLAY + REC
2) Decid vuestros nombres y la fecha
3) Explicad vuestros textos
4) Preguntad sobre el texto de vuestro compañero
5) ¡No paréis el magnetófono, por favor!

Figura 2. Instrucciones para grabar el intercambio de información

Mientras las díadas realizaban esta subtarea, las profesoras controlaban el progreso de la misma y ayudaban a los alumnos con la lengua meta, el inglés, si estos se lo pedían. Las profesoras siempre se dirigieron a los alumnos en inglés.

$\mathrm{Al}$ acabar la tarea 1, se archivó muestras de los materiales utilizados en la misma, las notas de campo y las grabaciones. Debido a un problema técnico, las grabaciones correspondientes a la primera subtarea no pudieron transcribirse. La segunda se convirtió así en el principal conjunto de datos del corpus.

\section{Análisis preliminar}

Los datos conversacionales se analizaron con el objetivo de descubrir qué actividades realizaban las parejas durante su interacción. Conocer estas actividades resultaba necesario para poder elaborar la definición de actividad on de los informantes, teniendo en cuenta la definición de tarea escolar desde la 
perspectiva del alumno propuesta por Bygate, Skehan y Swain (2001). La definición resultante es la siguiente:

Las parejas realizan una actividad on cuando:

1) graban su nombre y la fecha

2) demandan o proporcionan información sobre la selva, utilizando

a. la información contenida en los textos proporcionados para la realización de la subtarea precedente

b. sus conocimientos previos sobre el tema

3) negocian significados sobre la selva

4) trabajan en secuencias metalingüísticas para comunicar significados en la lengua meta

5) gestionan la tarea para

a. negociar el paso a una nueva fase de la misma

b. negociar el abandono o la continuación de la subtarea

c. decidir cuándo pedir ayuda a las profesoras u otras

6) evalúan parejas

d. su propia actuación

e. la de su compañero/-a

Figura 3. Definición de actividad on utilizada en este estudio

La definición de la figura 3 está compuesta por tres clases de actividades on. La primera es la conversación sobre el tema meta, relacionada con los apartados 1-4.

La segunda clase es la gestión de la interacción, que corresponde al apartado 5. La última clase, vinculada al apartado 6, es la evaluación.

En el apartado sexto de la definición no se incluyó ningún subapartado sobre la evaluación de la subtarea consistente en el intercambio de información o la evaluación de toda la tarea 1 porque ninguna pareja expresa su opinión sobre ellas. Sin embargo, la formulación de la definición no hubiera sido posible observando únicamente las actividades llevadas a cabo por los informantes. Conocer las instrucciones que recibieron también era necesario. El hecho de que, por ejemplo, los alumnos dijesen su nombre y la fecha al empezar la grabación se pudo clasificar como una actividad on porque esta constituía un intento de iniciar el desarrollo de la subtarea siguiendo las instrucciones recibidas. Por otro lado, no ceñirse a las instrucciones que les incitaban a centrarse en la información contenida en los textos aprendidos en la subtarea precedente no se consideró un abandono de la subtarea, si las parejas conversaban sobre algún otro tema 
relacionado con la selva o el Amazonas, es decir, si adaptaban la tarea a sus necesidades o intereses. Tampoco se consideró como actividades off aquellas en las que los alumnos recurrieron a estrategias como la traducción o la alternancia de códigos para conseguir comunicar significados relacionados con la selva, ya que los alumnos en contextos de contacto de lenguas desarrollan una consciencia metalingüística que les ayuda a usar sus L1 para rellenar las lagunas de las áreas de la lengua meta que todavía no han adquirido (Guasch y Milian 1999).

A continuación, se muestran fragmentos transcritos de la interacción que motivó la formulación de cada apartado de la definición ${ }^{6}$ (Tabla 2) y la simbología de transcripción utilizada en este estudio (Tabla 1).

Tabla 1. Simbología de transcripción utilizada en este estudio, propuesta por el equipo de investigación CLIL-SI

\begin{tabular}{|l|l|}
\hline Secuencias tonales: & Fragmentos incomprensibles: \\
Descendente । & Sintagma (xx) \\
Ascendente / & Oración (xxx) \\
Pausas: & Comentarios del transcriptor: \\
Corta (.) & $(($ texto)) \\
Larga (número de segundos) & Fragmentos emitidos mientras se ríe: \\
Alargamiento silábico: & ((ríe) (texto) \\
: & Texto en catalán: \\
Interrupciones: & Texto \\
Text- & Texto en español: \\
Intensidad: & Texto \\
Piano ${ }^{\circ}$ texto $^{\circ}$ & Traducción: \\
Forte TEXTO & \&Estu.: \\
\hline
\end{tabular}

Tabla 2. Definición de actividad on con fragmentos de interacción a modo de ejemplo

\begin{tabular}{|c|c|}
\hline Definición & Ejemplo \\
\hline $\begin{array}{l}\text { Las parejas realizan } \\
\text { actividades on cuando: }\end{array}$ & \\
\hline $\begin{array}{l}\text { 1) graban su nombre y la } \\
\text { fecha }\end{array}$ & $\begin{array}{l}\text { Pareja 8, turno 1: } \\
\text { Estudiante A: hello } \backslash \text { my name is ((nombre del alumno)) and today } \\
\text { is fourteen of march (1.0) fourteenth- fourteenth of march of two- } \\
\text { no } \backslash \text { twenty-two thousand/ } \\
\text { \&Estu. A: hola } \backslash \text { mi nombre es ((nombre del alumno)) y hoy es } \\
\text { catorce de marzo (1.0) catorce- catorce de marzo de dos- no } \\
\text { veintidós mil/ }\end{array}$ \\
\hline 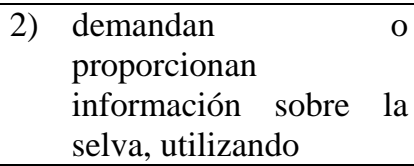 & \\
\hline
\end{tabular}




\begin{tabular}{|c|c|c|}
\hline \multicolumn{2}{|r|}{ Definición } & Ejemplo \\
\hline & $\begin{array}{l}\text { a. la información } \\
\text { contenida en los } \\
\text { textos } \\
\text { proporcionados para } \\
\text { la realización de la } \\
\text { subtarea precedente }\end{array}$ & $\begin{array}{l}\text { Pareja 6, turno 6: } \\
\text { Estudiante A: the rainforest is dense/ warm/ and wetl ((ríe)) } \\
\text { \&Estu. A:La selva es densa/cálida/ y húmeda ((ríe)) }\end{array}$ \\
\hline & $\begin{array}{l}\text { b.sus conocimientos } \\
\text { previos sobre el } \\
\text { tema }\end{array}$ & $\begin{array}{l}\text { Pareja 1, turnos 77-84: } \\
\text { Estudiante A: there are a lot of types of animals } \backslash \text { there are/ } \\
\text { \&Estu. A:Hay un montón de tipos de animales } \backslash \text { hay/ } \\
\text { Estudiante B: per example/ } \\
\text { \&Estu. B:Por ejemplo/ } \\
\text { Estudiante A: ((riendo)) (tucans)/ frogs/ (1.0) snakes/ } \\
\text { \&Estu. A:((riendo)) (tucanes)/ ranas/ (1.0) serpientes/ } \\
\text { Estudiante B: but we have got frogs herel } \\
\text { \&Estu. B:Pero tenemos ranas aquí } \\
\text { Estudiante A: yes } \backslash \text { but ((riendo)) (are di-) } \\
\text { \&Estu. A:Síl pero ((riendo)) (son di-) } \\
\text { Estudiante B: ((ríe)) } \\
\text { Estudiante A: are different than here } \\
\text { \&Estu. A:Son diferentes a las de aquí }\end{array}$ \\
\hline & $\begin{array}{l}\text { negocian significados } \\
\text { sobre la selva }\end{array}$ & $\begin{array}{l}\text { Pareja 6, turnos 16-20: } \\
\text { Estudiante A: ((ríe)) and/ ok\and then there is the contamination of } \\
\text { the oxygen on the earth } \\
\text { \&Estu. A:y/ ok\y entonces hay la contaminación del oxigeno en la } \\
\text { tierra } \\
\text { Estudiante B: ((ríe)) } \\
\text { Estudiante A: contamination } \\
\text { \&Estu. A:contaminación } \\
\text { Estudiante B: contamination and/ } \\
\text { \&Estu. B:Contaminación y/ } \\
\text { Estudiante A: segur contamination/ } \\
\text { \&Estu. A:Seguro contaminación/ } \\
\text { Estudiante B: ((ríe)) and the planetum: with the oxygen or (xxx) } \\
\text { \&Estu. B:y el planeta um: con el oxigeno o (xxx) }\end{array}$ \\
\hline & $\begin{array}{l}\text { trabajan en secuencias } \\
\text { metalingüísticas para } \\
\text { comunicar significados } \\
\text { en la lengua meta }\end{array}$ & \begin{tabular}{l} 
Pareja 7, turno 36-39: \\
Estudiante A: bueno/ que in the Amazonia (xx) overexploited \\
\&Estu. A:Bueno/ que en el Amazonas (xx) sobreexplotadol \\
Estudiante B: what you- què vols dir amb què l'Amazònia (xx) \\
overexploited/ \\
\&Estu. B:Qué tu- qué quieres decir con que el Amazonas (xx) \\
sobreexplotado/ \\
Estudiante A: que hay mucha explotación de árboles\} $\\
{\text { Estudiante B: ah/okl }}$ \\
\hline & gestionan la tarea para & \\
\hline & $\begin{array}{l}\text { a. negociar el paso a } \\
\text { una nueva fase de la } \\
\text { misma }\end{array}$ & 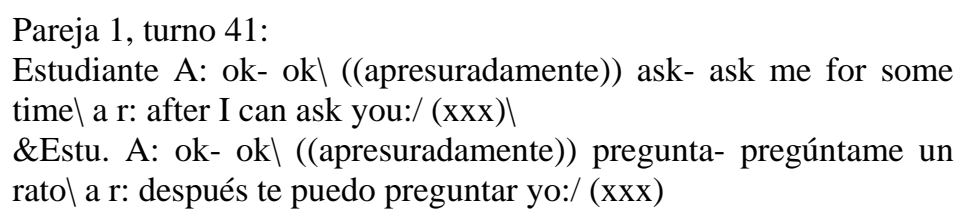 \\
\hline
\end{tabular}
\end{tabular}




\begin{tabular}{|c|c|}
\hline Definición & Ejemplo \\
\hline $\begin{array}{lll}\text { b. negociar } & \text { el } \\
\text { abandono o } & \text { la } \\
\text { continuación } & \text { de } & \text { la } \\
\text { subtarea } & & \end{array}$ & $\begin{array}{l}\text { Pareja 8, turnos 33-34: } \\
\text { Estudiante A: ok } \backslash \text { bye/ bye } \backslash \\
\text { \&Estu. A:Ok\adiós/ adiós } \backslash \\
\text { Estudiante B: NO } \backslash \text { NO STOP/ NO STOP } \backslash \\
\text { \&Estu. B:NO \NO PARES/ NO PARES } \backslash\end{array}$ \\
\hline $\begin{array}{l}\text { c. decidir cuándo } \\
\text { pedir ayuda a las } \\
\text { profesoras u otras } \\
\text { parejas }\end{array}$ & $\begin{array}{l}\text { Pareja 2, turno 37-8. } \\
\text { Estudiante A:((nombre de la profesora)) una pregunta/ com es diu } \\
\text { explica/ } \\
\text { \&Estu. A:((nombre de la profesora)) una pregunta/ Cómo se dice } \\
\text { explica/ }\end{array}$ \\
\hline 6) evalúan & \multirow{2}{*}{$\begin{array}{l}\text { Pareja 5, turno 13: } \\
\text { Estudiante A: Jo: (.) I you pain per haver-ho dit així } \\
\text { \&Estu. A:Jo: (.) yo tú pena por haberlo dicho así }\end{array}$} \\
\hline $\begin{array}{l}\text { a. su propia } \\
\text { actuación }\end{array}$ & \\
\hline $\begin{array}{l}\text { b. la actuación de su } \\
\text { compañero/-a }\end{array}$ & $\begin{array}{l}\text { Pareja 1, turno 102: } \\
\text { Estudiante A: }{ }^{\circ} \text { aha } l^{\circ} \text { so you understood well } \backslash \\
\text { \&Estu. A: }{ }^{\circ} \text { Ajá } \iota^{\circ} \text { así que entendiste bien } \backslash\end{array}$ \\
\hline
\end{tabular}

En lo concerniente a la actividad off, esta también se dividió entre actividad en la lengua meta y actividad en otras lenguas. La actividad en la lengua meta puede desarrollarse exclusivamente en dicha lengua o incluir otras, como es el caso del fragmento que ilustra esta actividad en la siguiente tabla.

Tabla 3. Clasificación de las actividades off y fragmentos de interacción a modo de ejemplo

\begin{tabular}{|c|c|}
\hline Tipo de actividad off & Ejemplo \\
\hline Uso de la lengua meta & $\begin{array}{l}\text { Pareja 9, turno 31: } \\
\text { Estudiante A: ((RÍE)) El Futbol Club Barcelona ((imitando la } \\
\text { pronunciación inglesa)) playing football contra versus Benfica. } \\
\text { Juventus versus Milan ((RÍE)) } \\
\text { \&Estu. A: ((RÍE)) El Fútbol Club Barcelona ((imitando la } \\
\text { pronunciación inglesa)) jugando a fútbol contra contra el Benfica. } \\
\text { El Juventus contra el Milán ((RÍE)) }\end{array}$ \\
\hline $\begin{array}{l}\text { Uso exclusivo de otras } \\
\text { lenguas }\end{array}$ & $\begin{array}{l}\text { Pareja 3, turno 42: } \\
\text { Estudiante A: ((ríe)) Saluda a la cámara/ saluda a la cámaral } \\
\text { ((ríe)) } \\
(2)\end{array}$ \\
\hline
\end{tabular}

La clasificación de los datos conversacionales de la manera expuesta aquí sirvió de base para la cuantificación de dichos datos. Tomando el segundo como unidad de medida ${ }^{7}$, se cronometró el tiempo que las parejas invirtieron en la conversación 
sobre el tema meta, la gestión de la interacción, la evaluación de la misma, actividad off en lengua meta y actividad off en otras lenguas. Posteriormente, se calculó los porcentajes para cada tipo de actividad. Los resultados cuantitativos obtenidos de esa manera se muestran en el siguiente apartado.

\section{Resultados}

Las tablas 4-6 contienen la información necesaria para contestar las preguntas de investigación. La tabla 4 encierra las cifras para responder a cuánto tiempo dedicaron los alumnos a realizar la subtarea del intercambio oral de información sobre la selva.

Tabla 4. Tiempo total de grabación y tiempos on y off

\begin{tabular}{|c|c|c|c|c|c|}
\hline \multirow[t]{2}{*}{ Pareja } & \multirow{2}{*}{$\begin{array}{c}\text { Tiempo total de } \\
\text { grabación } \\
\text { min. y s. }\end{array}$} & \multicolumn{2}{|c|}{$\begin{array}{c}\text { Tiempo dedicado a } \\
\text { actividades on }\end{array}$} & \multicolumn{2}{|c|}{$\begin{array}{l}\text { Tiempo dedicado a } \\
\text { actividades off }\end{array}$} \\
\hline & & min. y s. & $\%$ & min. y s. & $\%$ \\
\hline 1. & 6’47', & 6'9" & 90.66 & $38 ”$ & 9.34 \\
\hline 2. & 4'56', & 4’52” & 98.65 & $4 ”$ & 1.35 \\
\hline 3. & 6’31” & 5’30”' & 84.40 & 1'1" & 15.60 \\
\hline 4. & 4’24”' & 4'19”' & 98.11 & $5 "$ & 1.89 \\
\hline 5. & 2’58” & 2’58”' & 100 & 0 & 0 \\
\hline 6. & 3’40” & 3’40” & 100 & 0 & 0 \\
\hline 7. & 4’21”' & 4'16”' & 98.08 & $5 ”$ & 1.92 \\
\hline 8. & 3’46”' & 3’45”' & 99.56 & $1 ”$ & 0.44 \\
\hline 9. & 3’18” & 1’12” & 36.34 & 2'6"' & 63.63 \\
\hline 10. & 2’46” & 2’46” & 100 & 0 & 0 \\
\hline 11. & 5,43 & 5’35” & 97.67 & 8” & 2.33 \\
\hline Media & 4’28”' & 4'6" & 91.23 & $23 ”$ & 8.77 \\
\hline
\end{tabular}

Como se muestra en la tabla 4, el tiempo de grabación se sitúa entre los 2’46’' de Pareja 10 y los 6’47'’ de Pareja 1. Como ocurre con Pareja 2, Pareja 3 y Pareja 11, el tiempo de Pareja 1 es muy superior a la media (4’28’'). La media, a su vez, supera las expectativas de la profesora y las observadoras participantes sobre la duración de la actividad. 
La información de la tabla 4 también permite conocer cuánto tiempo emplean los alumnos en actividades on y off. Pareja 9 sólo se dedicó a actividades on durante el 36.34\%. Son los únicos que pasan la mayor parte de su tiempo en actividades off. La pareja que los sigue en porcentaje de actividades on, Pareja 3, estuvo ocupada con la subtarea durante casi todo su tiempo. Pareja 5, 6 y 10 pasaron en modo on el $100 \%$ del tiempo.

Las cifras del uso de la lengua meta durante actividades off se encuentran en la tabla 5.

Tabla 5. Actividad off en la lengua meta

\begin{tabular}{|c|c|c|c|}
\hline \multirow{2}{*}{ Pareja } & Tiempo dedicado a actividades off en lengua meta & $\begin{array}{c}\text { Tiempo dedicado } \\
\text { a actividades off }\end{array}$ \\
\cline { 2 - 4 } & min. y s. & \% tiempo off & $38 ”$ \\
\hline 1. & $5 ”$ & 13.16 & $4 ”$ \\
\hline 2. & $2 ”$ & 50 & 1 '1” \\
\hline 3. & $6 ”$ & 15.79 & $5 ”$ \\
\hline 4. & 0 & 0 & 0 \\
\hline 5. & 0 & 0 & 0 \\
\hline 6. & 0 & 0 & $5 ”$ \\
\hline 7. & $2 ”$ & 40 & $1 ”$ \\
\hline 8. & $1 ”$ & 100 & 2 '6” \\
\hline 9. & $93 ”$ & 73.81 & 0 \\
\hline 10. & 0 & 0 & $8 ”$ \\
\hline 11. & 0 & 0 & $23 ”$ \\
\hline Media & $10 ”$ & 26.61 & \\
\hline
\end{tabular}

Como se puede apreciar en la tabla 4, sólo dos de los ocho equipos con tiempo off, Pareja 4 y Pareja 11, no utilizan el inglés. Los seis equipos restantes dedican porciones muy diversas de su tiempo off a interactuar en esta lengua, entre el 13.16\% y 73.81\%. El porcentaje más alto es el de Pareja 9, quienes se mantienen off durante la mayor parte de su tiempo de grabación.

La tabla 6 contiene la información sobre los tres subtipos de actividad on: gestión y conversación sobre el tema meta y evaluación. 
Tabla 6. Tipos de actividad on

\begin{tabular}{|c|c|c|c|c|c|c|c|c|c|}
\hline \multirow{3}{*}{ 苞 } & \multicolumn{9}{|c|}{ Tiempo dedicado a actividades on } \\
\hline & \multicolumn{3}{|c|}{ Gestión } & \multicolumn{3}{|c|}{ Conversaciónsobre el tema } & \multicolumn{3}{|c|}{ Evaluación } \\
\hline & $\begin{array}{c}\min . y \\
\text { s. }\end{array}$ & $\begin{array}{c}\% \% \\
\text { tiempo } \\
\text { on }\end{array}$ & $\begin{array}{c}\% \% \\
\text { tiempo } \\
\text { total }\end{array}$ & $\begin{array}{c}\min . y \\
\text { s. }\end{array}$ & $\begin{array}{c}\% \\
\text { tiempo } \\
\text { on }\end{array}$ & $\begin{array}{c}\% \\
\text { tiempo } \\
\text { total }\end{array}$ & $\begin{array}{c}\min . \mathbf{y} \\
\text { s. }\end{array}$ & $\begin{array}{c}\% \\
\text { tiempo } \\
\text { on }\end{array}$ & $\begin{array}{c}\% \\
\text { tiempo } \\
\text { total } \\
\end{array}$ \\
\hline 1. & $51 ”$ & 13.82 & 12.53 & 5’13” & 84.82 & 76.90 & $5 ”$ & 1.36 & 1.23 \\
\hline 2. & $40 ”$ & 13.70 & 13.51 & $252 ”$ & 86.30 & 85.14 & 0 & 0 & 0 \\
\hline 3. & 3’35” & 65.15 & 54.99 & $61 ”$ & 34.85 & 29.41 & 0 & 0 & 0 \\
\hline 4. & 1’22” & 31.67 & 31.06 & $2 ’ 55 ”$ & 67.57 & 66.29 & $2 ”$ & 0.76 & 2.65 \\
\hline 5. & $47 ”$ & 26.40 & 26.40 & 2’4” & 69.66 & 69.66 & $7 ”$ & 3.94 & 3.94 \\
\hline 6. & $30 ”$ & 13.64 & 13.64 & 190” & 86.36 & 86.36 & 0 & 0 & 0 \\
\hline 7. & 1'8”' & 26.56 & 26.05 & $281 ”$ & 73.44 & 73.95 & 0 & 0 & 0 \\
\hline 8. & 1’19”' & 35.11 & 34.96 & 2’23”' & 63.56 & 63.27 & 3” & 1.33 & 1.33 \\
\hline 9. & $11^{\prime}$ & 18.03 & 5.56 & $50 ”$ & 81.97 & 25.25 & 0 & 0 & 0 \\
\hline 10. & $41^{\prime}$ & 24.70 & 24.70 & 2’5” & 75.30 & 75.30 & 0 & 0 & 0 \\
\hline 11. & 1'4"' & 19.10 & 18.66 & $271 ”$ & 80.90 & 79 & 0 & 0 & 0 \\
\hline $\begin{array}{l}\text { Me- } \\
\text { dia }\end{array}$ & 1'6”' & 26.17 & 23.82 & $3^{\prime}$ & 66.24 & 54.40 & $1.55 ”$ & 0.67 & 0.83 \\
\hline
\end{tabular}

La tabla 6 muestra que sólo cuatro equipos emplean tiempo en la evaluación y que las cifras alcanzadas por estos son inferiores al 4\%. Respecto a la gestión, todas las díadas le dedican tiempo, pero sólo Pareja 3 invierte la mitad de su tiempo on. Después de estos y de Pareja 9, cuyo porcentaje más alto es el de tiempo off, la díada con la cifra de conversación sobre el tema más baja es Pareja 8, quien le dedica el $63.27 \%$ de su tiempo total de grabación. La cifra más alta es la 
conseguida por Pareja 6, el 86.36\% del total. Por lo tanto, la conversación sobre el tema es la actividad a la que casi todas las díadas dedican la mayor parte del tiempo durante la ejecución de la subtarea.

\section{Discusión}

La subtarea oral en díada estudiada fue diseñada para promover el aprendizaje cooperativo de contenidos sobre la selva y el discurso científico necesario para transmitirlos. Para lograr este aprendizaje, los alumnos tienen que conversar sobre la selva durante el tiempo en que los docentes les ceden el control de la tarea. Conversar sobre el tema es precisamente lo que hacen nueve de las once díadas estudiadas durante gran parte del tiempo de grabación. Esto es, casi todos los alumnos se implican en la actividad conversacional meta aunque la profesora y las observadoras participantes no están vigilándolos constantemente.

Aunque las profesoras no estén presentes constantemente, los magnetófonos sí. Es posible que las díadas transfieran a dichos aparatos las funciones de control y evaluación ejercidas tradicionalmente por los docentes (Escobar y Nussbaum 2002; Nussbaum 1999). La presencia de los magnetófonos podría ser, pues, una razón que explicase el hecho de que casi todas las parejas invierten la mayor parte de su tiempo en trabajar sobre el tema meta. Escobar y Nussbaum (2008), tras estudiar la interacción de tres parejas mientras realizan la misma actividad, concluyen que los alumnos se esfuerzan en demostrar lo que saben, prestando un grado elevado de atención al discurso, para salvar así su imagen propia y la de su compañero. Esta conclusión es un argumento a favor de la concepción de los magnetófonos como una extensión de la figura docente.

Aparte de la presencia del magnetófono, pueden existir otros motivos por que la mayoría de las parejas se esfuerzan en interactuar entorno al tema meta. Uno de ellos sería que a los alumnos les resulte fácil entender qué se les pide en la tarea 1, ya que, en su diseño, se definieron unos estrechos márgenes de interacción por lo que respecta a la ejecución de la tarea, la lengua y la 
información sobre el tema. Otro sería el hecho de que la puntuación del test que sigue a la subtarea estudiada sea la media de la puntuación de cada miembro. Un último motivo es que la subtarea tiene un interés intrínseco para los alumnos. La autenticidad de contenido e interacción típicas de las tareas AICLE (Escobar 2004b) puede ser el origen de su interés y esfuerzo por realizarla. Indistintamente del origen de este interés, "si los estudiantes se implican en las tareas [...], es porque las encuentran útiles, agradables y relevantes” (Morgan-Fleming, Burley y Price 2003: 1). El interés de las díadas en la subtarea es la única razón que explicaría, además, por qué se ha obtenido un tiempo medio de grabación alto.

Con un comportamiento opuesto al exhibido por diez parejas, Pareja 9 decide abandonar la subtarea muy tempranamente y dedicarse a actividades off durante casi todo su tiempo de grabación. Durante el análisis preliminar de los datos, se observó un gran desajuste entre el nivel de dificultad de la subtarea y la Zona de Desarrollo Próximo (Vygotsky 1978) de esta pareja. Su negativa a intentar realizar el intercambio de información puede ser atribuida a dicho desajuste. Sin embargo, su comportamiento contrasta con el de otra pareja con un bajísimo nivel de competencias en inglés, Pareja 2, quienes desarrollan actividades on durante el $85.14 \%$ de su tiempo. Por consiguiente, las características intrínsecas de las díadas influyen en su comportamiento.

El comportamiento de Pareja 9 también pudo verse afectado por el contexto en el que les pide que lleven a cabo una subtarea de CCNN en inglés. El aula no es la de CCNN. La profesora presente tampoco es especialista en CCNN; es su profesora de inglés. En ese contexto, resulta posible abandonar la subtarea y reasignar el tiempo restante a practicar inglés, lo que justificaría el hecho de que Pareja 9 invirtiese casi todo su tiempo en interactuar en esa lengua. La motivación subyacente en la reasignación del tiempo restante por parte de Pareja 9 sería complacer a la profesora presente. Una profesora de inglés como LE, a diferencia de una profesora AICLE, podría alcanzar cierto grado de satisfacción con el rendimiento de esta díada durante el tiempo dedicado a actividades off. 


\section{Conclusión}

La primera impresión de las observadoras participantes fue que los alumnos habían estado perdiendo el tiempo en vez de trabajando en la subtarea asignada. No extraña, pues, que los docentes tengan sus reservas a cerca del fruto de las tareas orales en díada. Este estudio surge de la necesidad de explorar si este tipo de reservas están bien fundadas, contrastándolas con una cuantificación de la actividad on y off de una clase natural y diversa mientras ejecuta un intercambio oral de información. Los resultados del estudio no sólo sugieren que la mayoría de díadas inician actividades on y la mantienen mientras ejercen el control de la tarea, sino que también corroboran los resultados cualitativos obtenidos por Escobar y Nussbaum (2008) con una muestra del mismo corpus. Los resultados de ambos estudios contradicen la creencia de que las tareas en díada son improductivas. También la contradicen los resultados del estudio de tareas orales desde la perspectiva del alumno realizado por Masats, Nussbaum y Unamuno $(2007)^{8}$, entre otras publicaciones del grupo de investigación consolidada GREIP, cuyo interés investigador también se centra en la interacción entre educandos en Cataluña.

A pesar de que estudios como los citados en el párrafo anterior contradicen la intuición de los profesores, resulta imprescindible tener en cuenta sus creencias y sus necesidades al escoger temas de investigación sobre innovación docente y formas de evaluar dicha investigación. Por este motivo, investigadores universitarios del equipo CLIL-SI colaboraron con docentes en activo en el proyecto ArtICLE. Los investigadores se beneficiaron así de su saber hacer y obtuvieron acceso a experiencias de aula auténticas (Escobar 2008). Los docentes, a su vez, no sólo tuvieron el apoyo del equipo universitario durante la preparación

e implementación de la secuencia didáctica Rainforests. También pudieron 'comprobar los resultados de su trabajo de manera empírica y sistemática, cosa extremadamente valiosa para los profesores aunque infrecuentemente su alcance' 
(Ibíd. 137). Sin embargo, todavía está por demostrar si estas evidencias empíricas pueden llegar a modificar sus creencias y hábitos docentes a largo plazo.

\section{Bibliografía}

Aronson, E., Blaney, N., Sikes, J. \& Snapp, M. (1975). Busing and racial tension.The jigsaw route to learning and liking. Pyschology Today 8: 4350 .

Bygate, M., Skehan, P.\& Swain, M. (Eds.) (2001).Researching pedagogic tasks: second language learning, teaching and testing. London y New York: Longman.

Dalton-Puffer, C. (2007). Discourse in Content and Language Integrated Learning (CLIL) classrooms.Amsterdam: John Benjamin.

Escobar, C. (2004a). Para aprender a hablar hay que querer decir algo. Glosas Didácticas: Revista Electrónica Internacional 12(3): 81-90.

Escobar, C. (2004b). Content and language integrated learning: do they learn content? Do they learn language? In Anderson, J.D., Oro, J.M. \& Varela, J. (eds.) Linguistic perspectives from the classroom: language teaching in a multicultural Europe (pp. 839-850). Universidade de Santigo de Compostela.

Escobar, C. (2008). Talking English to learn Science.A CLIL experience in Barcelona. In Dooly, M.\& Eastment, D. (Eds.) How we're going about it. Teachers' voices on innovative approaches to teaching and learning languages (pp. 154-169). Newcastle-upon-Tyne: Cambridge Scholar Press.

Escobar, C. y Nussbaum, L. (2002). ¿Es posible evaluar la interacción oral en el aula? En: Miquel, L. y Sans, N. (Eds.) (2010) Expolingua 2002: Didáctica del Español como Lengua Extranjera. Monográfico MarcoELE 10: 37-52. Visto el 14 de marzo de 2011 en http://marcoele.com/descargas/expolingua_2002.escobar-nussbaum.pdf

Escobar, C. y Nussbaum, L. (2008). Tareas de intercambio de información y procesos de aprendizaje en el aula AICLE. En: Camps, A. y Milian, M. (coords.) Miradas y voces. Investigaciones sobre la educación lingüística y literaria en entornos plurilingües (pp. 159-178). Barcelona: Graó.

Escobar, C.\& Sanchez, A. (2009).Language learning through tasks in a CLIL Science classroom. PortaLinguarum 11: 65-83.

Eurydice (2006).Content and Language Integrated Learning (CLIL) at school in Europe.Visto septiembre 2008 en http://www.eurydice.org/ ressources/eurydice/pdf/0_integral/071EN.pdf.

Gajo, L. (2007). Linguistic knowledge and subject knowledge: how does bilingualism contribute to subject development? The International Journal of Bilingual Education and Bilingualism 10(5): 563-581. 
Guasch, O. y Milian, M. (1999). De como hablando para escribir se aprende lengua. Textos, 20, 50-60.

Horrillo Godino, Z. (2006). A study of the on-task and off-task activity carried out during a CLIL task. Trabajo de fin de máster en formación del profesorado no publicado. Universidad Autónoma de Barcelona.

Horrillo Godino, Z. (2008a). Initial steps in the co-construction of a space of dialogue between an expert in foreign language pedagogy and an expert in social science pedagogy. A case study. Trabajo de fin de Máster en investigación en didáctica de la lengua y la literatura no publicado. Universidad Autónoma de Barcelona.

Horrillo Godino, Z. (2008b). Two teachers in the classroom: teaching maths in English.Comunicación presentada en TESOL-SpainConvention 2008. UEM: 9 Marzo.

Horrillo Godino, Z. (2009). A Study of the on-task and off-task activity carried out by teenage learners during a content and language integrated learning task. International Journal of Learning, 16(11): 113-136.

Horrillo Godino, Z. (2010). A case study of cross-curricular dialogue as a part of teacher education in the CLIL (Content and Language Integrated Learning) approach.Critical and ReflexivePractice in Education, 2: 4-17. Visto el 14 de marzo de 2010 en http://www.marjon.ac.uk/research/criticalandreflectivepracticeineducation/ volume2/Horillo\%20Godino\%20CRPE\%20v02.pdf

Horrillo Godino, Z. (2011). Formación del profesorado en el enfoque AICLE: apreciaciones informadas de la didáctica de las materias en las que el otro es experto. Un estudio de caso. En: Escobar, C., Evnitskaya, N., Moore, E.\&Patiño, A. (Eds.) AICLE/CLIL/EMILE. Educació plurilingüe: experiencias, research\&Politiques (pp. 41-56).Bellaterra: Servei de Publicacions de la Universidad Autónoma de Barcelona.

Hüttner, J. \& Rieder-Bünemann, A. (2007). The effect of CLIL instruction on children's narrative competence.View[s] 15(3): 41-46. Visto 25 Junio 2008 en http://www.univie.ac.at/Anglistik/Views_0703.pdf.

Jiménez, M. (2009). Aprenentatge integrat de continguts i llengües AICLE) i alumnes amb necessitats educatives especials. Un estudi de cas. Trabajo de fin de máster en investigación en didáctica de la lengua y la literatura no publicado. Universidad Autónoma de Barcelona.

Jiménez, M. y Bazoco, J. (2009). Ciencias en inglés: también para alumnado con Necesidades Educativas Especiales. En Cuadernos de Pedagogía. Tema del Mes: Aprender en inglés. Aprendizaje Integrado de Contenidos y Lengua AICLE. 395: 70-72. Monografía.

Kagan, S. (1985). Cooperative learning: resources for teachers. Riverside: University of California.

Lantolf, J.P. (2006). Sociocultural theory and L2. SSLA, 28: 67-109. 
Lew, M., Mesch, D., Johnson, D. \& Johnson, R. (1986). Components of cooperative learning: effects of collaborative skills and academic group contingencies on academic achievement and mainstreaming. Contemporary Educational Psychology, 11: 229-239.

Linares, A.\& Whitaker, R. (2007). Talking and writing in a foreign language in CLIL contexts: a linguistic analysis of secondary school learners of geography and history. Revista Española de Lingüística Aplicada, 1: 8391. Monografía.

Long, M.H. (1983).Native speaker/non-native speaker conversation and the negotiation of comprehensible input. Applied Linguistics 4(2): 127-141.

Lyster, R. (2007). Learning and teaching languages through content. A counterbalanced approach. Amsterdam: John Benjamins.

Marsh, D., Maljiers, A.\&Hartiala, A. (2001). Profiling European CLIL classrooms: language opens doors. ER-Paino: University of Jyväskylä \& European platform for Dutch education.

Masats, D. (2008). El discurs dels aprenents d'anglès com a llengua estrangera: una aproximació interactivista al procés de construcció de tasques comunicatives. Tesis doctoral en didáctica de la lengua y la literatura no publicada. Universidad Autónoma de Barcelona.

Masats, D., Nussbaum, L.\& Unamuno, V. (2007). When activity shapes the repertoire of second language learners.En: Roberts, L. et al. (eds.) EUROSLA yearbook (selected papers of EUROSLA 15 \&16 conferences), 7(1): 121-147.

Mondada, L.\& Pekarek-Doehler, S. (2004). Second language acquisition as situated practice: task accomplishment in the French second language classroom. The Modern Language Journal 88(4): 501-518.

Morgan-Fleming, B., Burley, H.\& Price, M. (2003) The benefits of being off-task. International Journal of Social Education 18(1): 1-8.

Nunan, D. (1989). Designing tasks for the communicative classroom. Cambridge: CUP.

Nussbaum, L. (1999). Emergence de la conscience linguistique en travail de groupe entre apprenants de langue étrangère. Langages, 134: 35-50.

Skehan, P. (1998). Task-based instruction. Annual Review of Applied Linguistics 18: 268-286.

Slavin, R. (1984). Students motivating students to excel: cooperative incentives, cooperative tasks and student achievement. The Elementary School Journal, 85(1): 53-63.

Snow, M.A., Met, M. and Genesse, F. (1989).A conceptual framework for the integration of language and content in second/foreign language instruction. TESOL Quarterly 23(2): 201-209.

Vygotsky, L.S. (1978). Mind in society: the development of higher psychological processes. Cambridge: Harvard University Press. 


\section{Watson, S.B. (1992). The essential elements of cooperative learning.DigitalCommons@LibertyUniversity. Visto en octubre 2010 en http://digitalcommons.liberty.edu/educ fac pubs/10 \\ Zuengler, J.\&Miller, E.R. (2006).Cognitive and sociocultural perspectives: two parallel SLA worlds? TESOL Quarterly 40(1): 37-38.}

\section{Notas}

1 Asignaturas de contenido son aquellas materias que no se etiquetan como clases de lengua. La tradición reserva dicha etiqueta sólo para las clases en las que una L1, L2 o LE es, a la vez, la materia designada y el contenido de la interacción (Dalton-Puffer 2007).

2 Acrónimo del proyecto "Tareas colaborativas y aprendizajes lingüísticos y académicos en las aulas AICLE inclusivas de ciencias en lengua extranjera” (ARIE 2004-210060 y 2005-ARIE10056), financiado por el Departamento de Universidades, Investigación y Sociedad de la Información de la Generalidad de Cataluña.

3 Los materiales de la secuencia pueden consultarse en http://grupsderecerca.uab.cat/clilsi/

4 Por motivos de economía lingüística, de aquí en adelante se utilizará el término actividad on u on para referirse a cualquier actividad dirigida a la realización de la tarea. Asimismo, se aplicará el término actividad off u off a cualquier actividad que implique el abandono de esa tarea.

5 La inclusión de estos dos puntos en las definiciones (la finalidad comunicativa y la orientación hacia la consecución de un objetivo) es lo que, en definitiva, distingue a una tarea de un ejercicio, como, por ejemplo las conversiones gramaticales, y de una actividad (Escobar 2004a), como, por ejemplo, indicar un camino en LE practicando ciertas estructuras.

6 Véase HorrilloGodino (2006) para consultar las transcripciones íntegras y la división de estas en actividades on y off.

7 Para medir períodos de tiempo con exactitud, tener entradas (indicaciones) en las transcripciones se convirtió en algo imprescindible. Por esta razón, se resaltó con colores los puntos en los que cualquier tipo de actividad on u off empieza y acaba.

8 Masats, Nussbaum y Unamuno (2007) estudia tres díadas que representan tres estadios diferentes de desarrollo de las competencias necesarias para interactuar en inglés como LE. Aunque cada díada trabaja en una tarea distinta, las tres recurren a la alternancia y mezcla de códigos, sustitución léxica y paráfrasis para pedir ayuda o asegurarse de que han comprendido correctamente un significado en su empeño por cumplir con las demandas de la tarea.

\section{Referencias de la autora:}

Zoraida HorrilloGodino, tras licenciarse en Filología Inglesa, obtuvo un Máster en Formación Inicial del Profesorado y un Máster en Investigación en Didáctica de la Lengua y la Literatura. Trabajó como investigadora en el Departamento de Psicología y Ciencias de la Educación de la Universidad Abierta de Cataluña (UOC) y en el grupo de investigación consolidada GREIP (Grupo de Investigación en Educación e Interacción Plurilingüe) de la Universidad Autónoma de Barcelona (UAB). Actualmente sigue colaborando con uno de los equipos de investigación de GREIP, CLIL-SI, especializado en la semiimmersión en lengua extranjera en el aula. Además, ejerce como docente en diferentes niveles educativos.

\section{Email: zhorrillo@gmail.com}

Bellaterra Journal of Teaching \& Learning Language \& Literature. 4.2

(May-June 2011): 71-95. ISSN 2013-6196. 
Agradecimientos: Quiero expresar aquí mi gratitud a todos los que han hecho posible este estudio: los informantes, tanto los alumnos como la profesora; las observadoras participantes y demás miembros del equipo de investigación CLIL-SI que participaron directa o indirectamente en la investigación; las revisoras anónimas de este artículo, cuyas detalladas observaciones me han resultado muy útiles; y la Dra. Cristina Escobar, quien me ha brindado apoyo y consejo desde que inicié el trabajo de final de máster en que se basa el presente estudio. 